\title{
Stimulus variables affecting induced rotation
}

\author{
BARRY MAPPERSON \\ University of Tasmania, Hobart, Tasmania, Australia \\ and \\ WILLIAM LOVEGROVE \\ University of Wollongong, Wollongong, New South Wales, Australia
}

\begin{abstract}
Dunker (1929/1938) reported that a stationary pattern surrounded by a rotating annulus appears to rotate in the opposite direction. Our experiments show that this rotary induced motion is affected by varying the velocity, pattern, and contrast of the inducing stimulus, is unaffected by the test-stimulus pattern, and is affected by test-stimulus contrast only at low contrast levels.
\end{abstract}

Dunker (1929/1938) reported that a stationary pattern would seem to rotate in the direction opposite to the actual rotation of a surrounding annulus. No subsequent direct investigation of this effect appears to have been carried out until the recent work of Day (1981) and Reinhardt-Rutland $(1981,1983 b)$. In the present paper, we investigate specific issues arising from previous work and, more generally, examine the effect on rotary induced motion of variables known to influence the motion aftereffects.

\section{EXPERIMENT 1}

Most direct and indirect investigations of rotary induced motion have involved very low velocities of rotation, perhaps as a result of the statement by Dunker $(1929 / 1938$, p. 166) that "the phenomenon of induced motion in the smaller disk was diminished or cancelled ... by increasing the speed of the larger disk." Precisely what is meant here is unclear, since a footnote on the same page states that "at speeds above $5^{\circ}$ per sec the induced motion, although greater, became less intense" (emphasis added). Day (1981) found no significant change in the frequency with which induced rotation was reported as the inducing velocity was increased from $4 \% \mathrm{sec}$ to $15 \%$ sec, and Mayhew (1975, p. 326), in an experiment in which a rotating dot pattern was superimposed on a stationary one, reported "pronounced induced motion" with an angular velocity of $30 \% / \mathrm{sec}$.

It was nevertheless with some surprise that we found in preliminary trials that substantial induced motion occurred even when the inducing stimulus was rotating at several hundred degrees per second. In the following experiment, therefore, we investigated the behavior of rotary motion induced by high velocities, using a range of stimulus patterns. The importance of this latter variable

Correspondence may be sent to William Lovegrove, Department of Psychology, The University of Wollongong, P.O. Box 1144, Wollongong, NSW 2500, Australia. is that for the aftereffects of rotary motion, the optimal velocity for producing any aftereffect depends on the periodicity of the pattern used, and it is best expressed in terms of the pass rate-that is, the number of elements passing a given point per unit of time (see Pantle, 1974). As a result, an interaction between the inducing velocity and the stimulus pattern would be expected.

\section{Method}

Subjects. There were 6 volunteer subjects in this and the following experiments.

Apparatus. The stimulus display, presented in a Scientific Prototype tachistoscope (Model GB), consisted of a circular test pattern $\left(1.3^{\circ} \mathrm{di}-\right.$ ameter) surrounded by a rotating annular inducing stimulus $\left(1.3^{\circ}\right.$ i.d., $5^{\circ}$ o.d.). The inducing and test patterns were always identical; they consisted of alternate black and white sectors equal in size. Patterns containing 8,16 , and 32 sectors were used. Their contrast was 0.6 , and their space-average luminance $9.2 \mathrm{ftL}$. The inducing stimulus was rotated by a variable electric motor, and the test stimulus could be manually rotated by the observer's turning a wheel in front of him.

Procedure. Observers were shown the stimulus display and told that their task was to keep the center portion perceptually stationary. Any apparent movement could be cancelled by manual contrarotation. Up to four 60 -sec practice trials were held for each condition, followed by six 60 -sec experimental trials. Within any one session this procedure was carried out for four velocities of inducing movement: $100^{\circ}, 200^{\circ}$, $300^{\circ}$, and $500^{\circ} / \mathrm{sec}$. These velocities were presented in a different randomized order for each subject. All observers participated in three test sessions, one for each pattern. The order of presentation of patterns was counterbalanced across subjects.

\section{Results and Discussion}

The mean amounts of induced motion in each of the twelve experimental conditions are shown in Figure 1. An analysis of variance showed a significant effect of inducing-stimulus velocity $[F(3,15)=5.0, p<.02]$. Subsequent Duncan tests showed that for all three patterns, the amount of movement induced by the $500 \% \mathrm{sec}$ stimulus was significantly $(p<.05)$ less than for either the $200 \% / \mathrm{sec}$ or the $300 \% \mathrm{sec}$ stimuli. For the 16 and 32 sector patterns it was also significantly less than that induced by the $100^{\circ} / \mathrm{sec}$ stimuli.

It will be noted that this decline in induced movement at the highest velocity used is particularly acute for the 32-sector stimulus. This stimulus was frequently described 


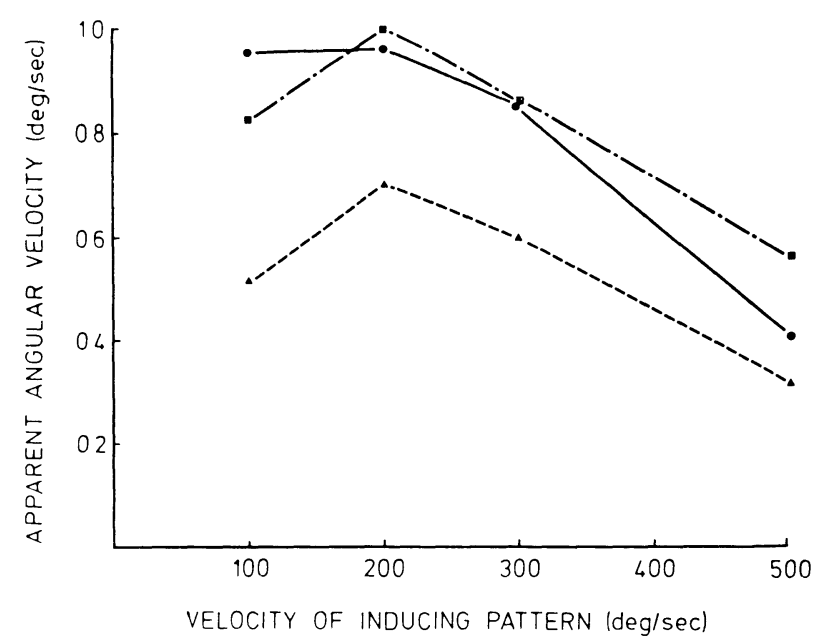

Figure 1. Mean angular velocities for cancellation of rotation induced by various surround velocities, with both patterns consisting of 8 sectors $(\Delta---\Delta), 16$ sectors $(\square---\square)$, or 32 sectors $(\bullet-\bullet)$.

as "blurring out" and becoming a flickering field with no clear direction of rotation.

The effect of pattern was below the level of significance $[F(2,10)=2.0, p>.05]$, although a significant effect might have been expected on the basis of previous work (Day, 1981). This may in part be due to this factor's acquiring high variability through being manipulated between sessions. It should be noted, however, that Duncan tests showed the induced movement for the 8-sector pattern to be significantly less than that for the 16-sector pattern at every velocity, and significantly less than that for the 32-sector pattern at all but the highest velocity.

Contrary to expectations based on aftereffect studies, there was no indication of an interaction between the velocity and pattern variables $[F(6,30)=1.0, p>.4)$. We conclude that substantial rotary motion can be induced with much greater velocities than have previously been reported; that the amount of motion is dependent on the inducing velocity, but that this relationship cannot be defined in terms of the pass rate.

\section{EXPERIMENT 2}

Day $(1981$, p. 497) discovered that progressively increasing the number of radial lines in the inducing stimulus from 2 to 16 produced a nonlinear increase in the amount of induced motion, and suggested that this "is due to the 'shearing' frequency of inner and outer lines. That is, the frequency with which outer lines shear in respect of inner lines determines apparent [induced] velocity up to a limiting frequency."

For all inducing stimuli, however, the test stimulus used was one with 16 radial lines. The increase in induced motion with increases from 2 to 16 in the number of lines in the inducing stimulus could therefore be simply attributed to the increase in pattern similarity between the inducing and test stimuli.
Both the "shearing-strength" and the "patternsimilarity" hypotheses can be tested by a factorial experiment in which the effect of each of several inducing stimuli is determined for the equivalent range of test stimuli. The pattern-similarity hypothesis would predict no main effects due to either inducing- or test-stimulus patterns as such, but a strong interaction between these factors. The shearing-strength hypothesis, in contrast, would predict that increasing the number of contours in either the test or the inducing stimulus would, up to a point, increase the shearing strength and hence the amount of induced motion. This hypothesis would also predict an interaction effect, since the effect of increasing the number of contours in the inducing stimulus will saturate earlier for test stimuli with many contours than for those with few.

\section{Method}

The method was identical to that described for Experiment 1, except that the inducing stimulus was always presented at a velocity of $300 \% \mathrm{sec}$, and the amount of motion created by each of the three inducing stimuli was measured on each of the three test stimuli. The inducing-stimulus pattern was varied within sessions, and the test-stimulus pattern was varied across sessions. The presentation orders of each variable were counterbalanced across subjects.

\section{Results and Discussion}

The mean amounts of induced motion under the nine conditions are shown in Figure 2. It will be evident that the results compel the rejection of both of the competing experimental hypotheses.

There is a significant effect of inducing-stimulus pattern $[F(2,10)=10.6, p<.005]$, as predicted by the shearing-strength hypothesis, but contrary to the patternsimilarity hypothesis. On the other hand, there is no corresponding main effect of test-stimulus pattern $[F(2,10)$ $=1.1, p>.05]-\mathrm{a}$ result that is in accord with the pattern-similarity hypothesis, but contrary to the shearing-

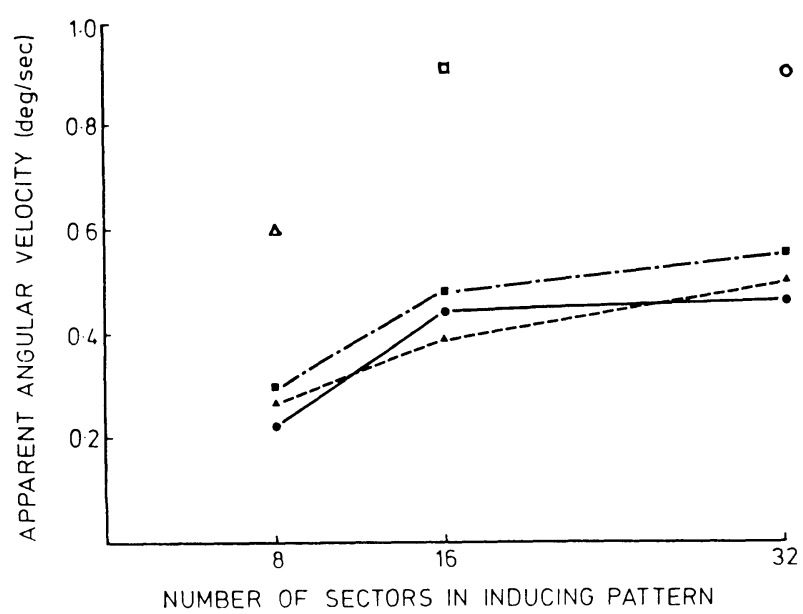

Figure 2. Mean angular velocities for cancellation of rotation induced by various surround patterns upon centers containing 8 sectors $(\Delta---\Delta), 16$ sectors $(\square---\square)$, or 32 sectors $(\bullet-\bullet)$. Corresponding data from Experiment 1 are plotted as hollow symbols. 
strength hypothesis. The total absence of any interaction effect $[F(4,20)=0.6, p>.6]$ is contrary to both hypotheses.

Several alternative explanations can also be eliminated. The increase in the number of sectors in the inducing stimulus increases both the pass rate and the apparent velocity, and it might be thought that the increased induced rotation is due to one of these factors. Both pass rate and apparent velocity are, however, also increased by simply increasing the actual velocity, and it will be recalled from Experiment 1 that, for all patterns, increasing the actual velocity of the inducing stimulus beyond that selected for this experiment did not increase induced motion but significantly decreased it. Thus although it is clear that increasing the number of moving elements in the inducing stimulus increases, up to a point, the induced motion, we are unable to explain why this is so.

It will be noted that three conditions in this experiment were identical to three conditions of Experiment 1, which have been included on Figure 2 in the form of open symbols. Although within each experiment the relative positions of the three points are similar, approximately twice as much movement was experienced by the group participating in Experiment 1. This surprisingly high degree of variability suggests that when considering this effect, one would be unwise to base any conclusions on merely quantitative differences between groups.

\section{EXPERIMENT 3}

Pantle, Lehmkuhle, and Caudill (1978) have summarized a quantity of data which shows that aftereffects of motion increase as the log contrast of the inducing stimulus increases, but only up to 5-6 times threshold, with further increases having little or no effect. The effect of test-stimulus contrast on the linear-movement aftereffect cannot, however, be fitted by any simple function (Keck, Palella, \& Pantle, 1976).

Experiment 3 was carried out to determine the effects on rotary induced motion of manipulating separately the contrast of the inducing and the test stimuli.

\section{Method}

Both the inducing and the test stimulus were provided by 16 -sector disks presented at a constant space-average luminance of $3.8 \mathrm{ftL}$. The inducing annulus was rotated at a constant velocity of $300 \% \mathrm{sec}$. The experiment was conducted in two sessions, in each of which either the inducing or the test stimulus was presented at a constance contrast $(0.85)$, whereas the other stimulus was presented at contrast levels of $0.1,0.2$, 0.4 and 0.8 . Contrast was varied while space-average luminance was held constant, by means of a procedure described in Richards (1971). In other aspects, the method was as described earlier.

\section{Results and Discussion}

The mean amounts of induced motion for the eight conditions are graphed in Figure 3. An analysis of variance disclosed that significantly more movement was induced by a constant high-contrast inducing stimulus upon a varied lower-contrast test stimulus than it was when these factors were reversed $[F(1,5)=9.2, p<.05]$. Overall,

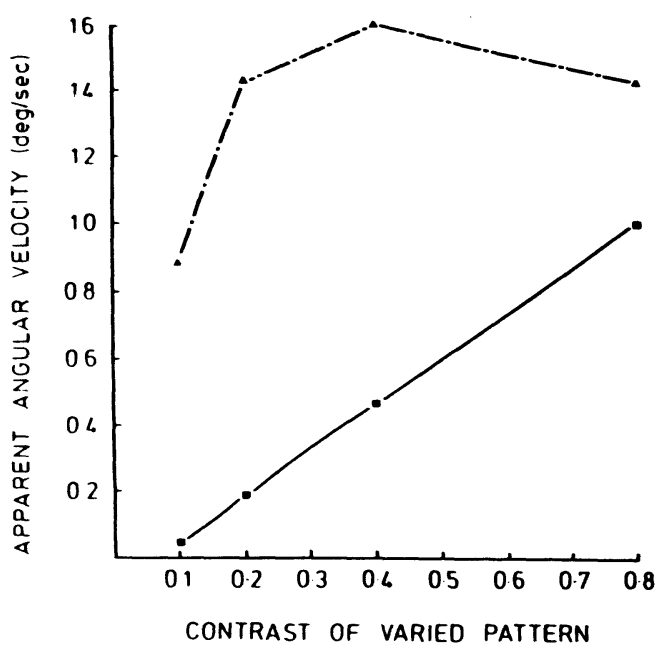

Figure 3. Mean angular velocities for cancellation of rotation induced with varying contrasts of inducing surround $(a-v)$ and of center $(\Lambda--\Lambda)$.

the effect of increasing contrast in either stimulus was to increase the amount of induced motion $[F(3,15)=11.8$, $p<.0005]$. A strong, though nonsignificant, interaction factor $[F(3,15)=2.9, .07>p>.06]$ suggests, however, that the effects of increasing contrast may differ somewhat for the inducing and the test stimuli. More detailed examination tends to support this. In the case of the inducing stimulus, the effect of contrast is remarkably consistent: for every observer, every increase in contrast resulted in an increase in the amount of induced rotation. The increase appears to be linear rather than logarithmic, and it clearly does not asymptote at the low levels expected from studies of the aftereffects of motion (Pantle et al., 1978). Although observations on linear motion suggest that the increasing contrast may have brought about some increase in the perceived velocity (Thompson, 1982), this is unlikely to be a relevant factor, for reasons discussed in the previous experiment.

Contrary, once again, to expectations based on the effects of test-stimulus contrast on the aftereffects of moving stimuli, increases in test-stimulus contrast tended to increase, rather than reduce, the amount of induced movement. The increase is due, however, entirely to the low amount of rotation reported on the lowest-contrast stimulus; the three higher contrasts show no significant differences.

Campbell and Maffei (1981) note that, at very slow rotational velocities, the perceived velocity increases as contrast increases, up to a point, and then it asymptotes. We tentatively suggest that this effect may also occur with the slow induced motion, reducing it below threshold at low contrasts but leaving higher contrast test stimuli unaffected.

\section{GENERAL DISCUSSION}

In the present experiments, we set out to examine whether variables known to influence the motion aftereffect produce similar effects upon 
induced rotation. In no case were similarities found between the simultaneous and successively induced effects of movement. This appears to create problems for attempts to explain the phenomena in terms of lateral inhibition between movement detectors (e.g., Reinhardt-Rutland, 1983a, 1983b).

A more general problem for this proposal is that although the inducing stimulation (and, presumably, any resultant lateral inhibition) is constant and continuous, the induced motion is not. This variation in the effect seems more compatible with approaches that stress the perceptual ambiguity of relative displacement (Day, 1981). Investigations of the more traditional linear form of Dunker-type induced movement have shown that the movement may be attributed to the moving stimulus, the stationary stimulus, or both stimuli simultaneously (e.g., Day, 1978; Day, Millar, \& Dickinson, 1979.) Although in the present case the inducing stimulus is always seen as moving, one might conceivably expect fluctuations to occur between the attribution of movement exclusively to the inducing stimulus, and to differential movement of both stimuli.

When large displays are rotated about the line of sight, observers feel their body tilted and experience vertical edges as tilted, opposite to the direction of rotation. This phenomenon, known as roll vection, is attributed to interactions between the visual and vestibular systems (see Dichgans \& Brandt, 1974, 1978 for summaries). Reinhardt-Rutland (1981) has already drawn attention to the fact that the peripheral location of the inducing stimulus is of great importance in both induced rotation and roll vection (Dichgans \& Brandt, 1974), but also notes that induced rotation is obtained under conditions where negligible roll vection would occur.

Although this is quite true, we were interested to note that on five occasions during the present series of experiments, observers reported disorientation, dizziness, and difficulty in walking, once the experiment was over. The most severely affected observer had to remain propped up between the corner of the walls and the floor for approximately $5 \mathrm{~min}$ before leaving the room. The same observer, however, reported no discomfort after a later session in which the experimental conditions were such that every observer experienced significantly less induced rotation. Such responses could result from prolonged, though mild, stimulation of the system that is involved in roll vection.

If the same system were to underly both roll vection and induced rotation, it would not be surprising that much stronger stimulation is required for the former. For roll vection to occur, the visual stimulation must overcome the directly competing input from vestibular and somatosensory receptors, whereas in induced rotation, competing cues would seem to be less direct and potent.

It is of interest that both induced rotation and roll vection "may appear to alternate between two states somewhat like reversals of figure and ground. In one state the motion is shared by both inner and outer fields, while in the second the inner field appears to stop rotating and only the outer field moves ... the tilt is also reduced ... and the sense of self-rotation ceases" (Held, Dichgans, \& Bauer, 1975, p. 358).

Unfortunately, the data from the present experiments cannot be compared with the data in studies of roll vection. Contrast has not been manipulated in roll vection studies, and there is insufficient overlap between the range of velocities used in the present experiments and those used in studies on roll vection (Dichgans, Held, Young, \& Brandt, 1972; Held et al., 1975; Hughes, Brecher, \& Fishkin, 1972) to permit any conclusions to be reached.

Brandt, Wist, and Dichgans (1975) have found that up to a point roll vection is increased as the number of randomly positioned moving dots is increased; then it asymptotes. These data cannot, however, be compared with the present and previous findings on the influence of the number of lines or sectors on induced rotation. As previously noted, roll vection is affected more by the peripheral location of moving stimuli than by the moving area. It would seem probable, therefore, that dots randomly allocated to the periphery of the annulus would have a major effect, while those randomly allocated to more central positions would have less influence. Thus the effective number of moving elements for optimal roll vection remains debatable.

The possibility of connections between induced rotation and roll vection cannot therefore be ascertained from existing data. On the basis of the present findings, however, it would appear that this may be a more fruitful line of investigation than attempts to equate simultaneous and successive effects of motion.

\section{REFERENCES}

BrandT, T., Wist, E. R., \& Dichgans, J. (1975). Foreground and background in dynamic spatial orientation. Perception \& Psychophysics, 17, 497-503.

Campbell, F. W., \& MafFeI, L. (1981). The influence of spatial frequency and contrast on the perception of moving patterns. Vision Research, 21, 713-721.

DAY, R. H. (1978). Induced visual movement as nonveridical resolution of displacement ambiguity. Perception \& Psychophysics, 23, 205-209.

DAY, R. H. (1981). Induced rotation with concentric patterns. Perception \& Psychophysics, 29, 493-499.

Day, R. H., Millar, J., \& Dickinson, R. G. (1979). Induced movement as nonveridical resolution of displacement ambiguity: Effect of enclosure and number of field elements. Perception \& Psychophysics, 25, 23-28.

DichGans, J., \& BrandT, T. (1974). The psychophysics of visually induced perception of self-motion and tilt. In F. O. Schmidt \& F. G. Worden (Eds.), The neurosciences: Third study program (pp. 123129). Cambridge, MA: MIT Press.

Dichgans, J., \& BrandT, T. (1978). Visual-vestibular interaction: Effects on self-motion perception and postural control. In R. Held, H. W. Leibowitz, \& H. L. Teuber (Eds.), Handbook of sensory physiology (pp. 755-804). Berlin: Springer.

Dichgans, J., Held, R., Young, L. R., \& Brandt, T. (1972). Moving visual scenes influence the apparent direction of gravity. Science, 178, 1217-1219.

DuNKER, K. (1938). Induced Motion. In W. D. Ellis (Ed. and Trans.), A source book of Gestalt psychology (pp. 169-172). London: Paul, Trench and Trubner. (Reprinted from Psychologische Forschung, 1929, 12, 180-259.)

Held, R., Dichgans, J., \& Bauer, J. (1975). Characteristics of moving visual scenes influence spatial orientation. Vision Research, 15 , 357-365.

Hughes, P. C., Brecher, G. A., \& Fishkin, S. M. (1972). Effects of rotating backgrounds upon the perception of verticality. Perception \& Psychophysics, 11, 135-138.

Keck, M. J., Palella, T. D., \& Pantle, A. (1976). Motion aftereffect as a function of the contrast of sinusoidal gratings. Vision Research, 16, 187-191.

MaYHEW, J. E. W. (1975). The effect of stationary patterns on adaptation for movement: Evidence for inhibitory interaction. Perception, 4, 311-329.

Pantle, A. (1974). Motion aftereffect magnitude as a measure of the spatio-temporal response properties of direction-sensitive analysers. Vision Research, 14, 1229-1236.

Pantle, A., Lehmkuhle, S., \& Caudill, M. (1978). On the capacity of directionally selective mechanisms to encode different dimensions of moving stimuli. Perception, 7, 261-267.

ReinhardT-Rutland, A. H. (1981). Peripheral movement, induced movement, and aftereffects from induced movement. Perception, 10, 173-182.

ReINHARDT-Rutland, A. H. (1983a). Aftereffect of induced rotation: Number of lines in inducing stimulus. Perceptual \& Motor Skills, 57, $39-42$.

Reinhardt-Rutland, A. H. (1983b). Aftereffect of induced rotation: Separation of inducing and static areas, and monocular component. Perceptual \& Motor Skills, 56, 239-242.

RICHARDS, W. (1971). Motion detection in man and other animals. Brain, Behavior \& Evolution, 4, 161-181.

Thомpson, P. (1982). Perceived rate of movement depends on contrast. Vision Research, 22, 377-380.

(Manuscript received November 8, 1988.) 